\title{
LIMONIIDAE ET PTYCHOPTERIDAE DU SUD-OUEST DE LA FRANCE [DIPTERA, NEMATOCERA]
}

( $2^{\mathrm{e}}$ note)

par A. G. B. THOMAS'.

240 imagos appartenant à 31 espèces de Limoniidae et une espèce de Ptychoptcridae sont citées de l'environnement immédiat de cours d'eau, jusqu'à $2200 \mathrm{~m}$ d'altitude. 21 espèces sont nouvelles pour la Faune de France, 5 autres pour celle des Pyrénées Françaises. Une synonymie est proposée.

Les larves de Pseudolimnophila sepium, Idiocera (Protogonomyia) limbata, Molophilus bifidus, $M$. flavus et Ptychoptera paludosa sont signalées pour la première fois dans des biotopes d'eau courante.

\section{Limoniidae and Ptychopteridae \\ from the south-west of France (Diptera: Nematocera).}

240 adults belonging to 31 species of Limoniidae and one species of Ptychopteridae were taken in the immediate vicinity of streams up to an altitude of $2,200 \mathrm{~m}$. 21 species are new records for the fauna of France, 5 others are new records for the French Pyrenees. A synonymy is proposed.

The larvae of Pseudolimnophila sepium, Idiocera (Protogonomyia) limbata, Molophilus bifidus, M. flavus, and Ptychoptera paludosa are recorded for the first time in a running-water biotope.

Si l'étude des "Tipuliformes" sensu lato a connu pendant ces quinze dernières années un développement important dans plusieurs pays d'Europe, la faune de la France demeure très incomplètement connue: le travail le plus important sur ce sujet restant celui de Pierre (1924)! D'autre part, d'un point de vue purement hydrobiologique, les larves d'un nombre élevé d'espèces, de Limoniidae en particulier, peuplent divers milieux aquatiques.

A partir de chasses d'imagos effectuées en bordure immédiate de torrents et de cours d'eau de plusieurs départements du Sud-Ouest, 48 espèces de Limoniidae et Ptychopteridae ont été citées précédemment (Thomas, 1968, 1972). La présente liste (32 espèces) en porte le nombre total provisoire à 80 . Les citations précédées de deux astérisques $\left({ }^{* *}\right)$ sont, à ma connaissance, nouvelles pour la France ; celles précédées d'un astérisque $\left(^{*}\right)$ sont nouvelles pour les Pyrénées françaises.

1. Laboratoire d'Hydrobiologie, Université Paul-Sabatier, 118, route de Narbonne, 31077 Toulouse Cedex, France. 
Les abréviations utilisées correspondent respectivement aux départements suivants (carte 1):

Ar : Ariège ; Av : Aveyron ; HG : Haute-Garonne ; HP : Hautes-Pyrénées ; LG : Lot-et-Garonne ; Lt : Lot ; Lz : Lozère ; T : Tarn.

Pour chaque espèce, les départements sont cités du Sud (Pyrénées) vers le Nord.

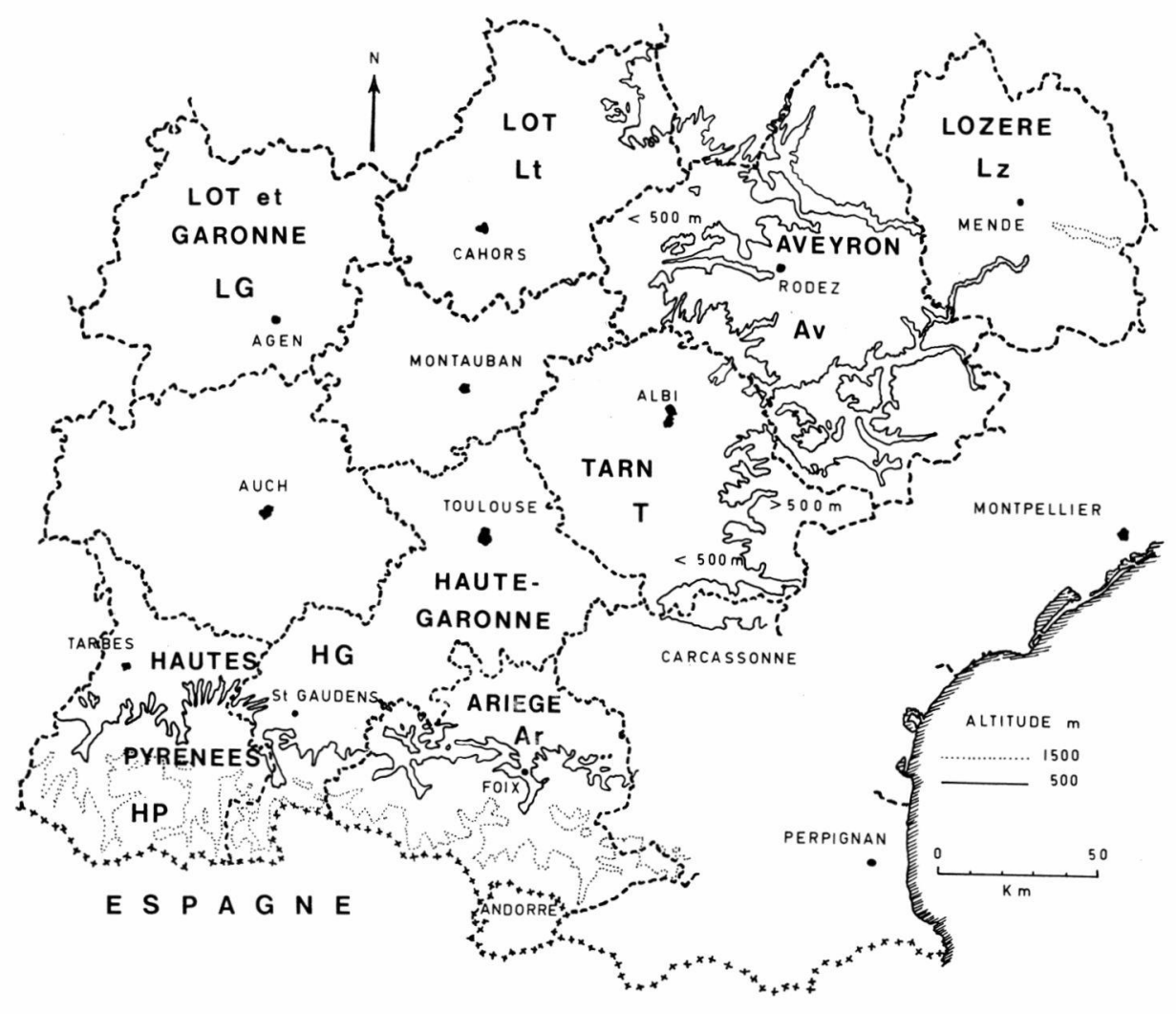

CARTE 1. - Le Sud-Ouest de la France: départements prospectés.

\section{LIMONIIDAE}

La classification des espèces de cette famille est actuellement l'objet de profonds remaniements. Les subvidisions proposées par Savtshenko et Krivolutskaya (1976) ont été reprises ici. A ce propos, 
j'ai plaisir à remercier M. le $\mathrm{D}^{\mathrm{r}} \mathrm{E}$. Savtshenko (Kiev) pour son avis sur l'appartenance générique de plusieurs espèces pyrénéennes.

\section{SOUS-FAMILLE DES PEDICIINAE}

\section{TRIBU DES PEDICIINI}

Genre Pedicia Latreille, 1809.

Sous-genre Crunobia Kolenati, 1859.

** 1. P. (C.) straminea (Meigen, 1838).

7 ô. HP : 1870 à $1180 \mathrm{~m}, 26-\mathrm{VI}$ à $18-\mathrm{IX}$.

\section{TRIBU DES ULINI}

Genre Ula Haliday, 1833.

** 2. U. (s. str.) bolitophila Loew, 1869.

3 ô. HP : $1000 \mathrm{~m}, 10$-VIII.

* 3. U. (s. str.) sylvatica (Meigen, 1818).

(= macroptera Macquart, 1826). 2 s. HP: $1180 \mathrm{~m}, 4-\mathrm{VIII}$; Ar: $310 \mathrm{~m}, 25-\mathrm{V}$.

\section{SOUS-FAMILLE DES HEXATOMINAE}

\section{TRIBU DES LIMNOPHILINI}

\section{Genre Pseudolimnophila Alexander, 1919.}

4. P. lucorum (Meigen, 1818).

16 ô, 9 ९. HP : 1250 à $710 \mathrm{~m}, 6$-VI à 18-VIII ; HG : $190 \mathrm{~m}, 25-\mathrm{V}$ et 4-VIII ; Lt : $145 \mathrm{~m}, 20$-IX.

* 5. P. sepium (Verrall, 1886).

12 ô, 2 q. HP 1050 à $1000 \mathrm{~m}, 29-\mathrm{VII}$ et 9-VIII ; Ar : $460 \mathrm{~m}, 1-\mathrm{VI}$; HG : $190 \mathrm{~m}, 25-\mathrm{V}$ à 2-VI et 4-VIII à 25-IX; Lt: $145 \mathrm{~m}, 20-\mathrm{IX}$.

Cette espèce ne figure pas dans la Limnofauna Europaea (Nielsen in Illies, 1967). Elle est signalée par Brindle et Bryce (1960) puis par Brindle (1967) dans les sols marécageux, généralement saturés d'eau, ou près des eaux stagnantes. En fait, elle peut se montrer plus franchement aquatique puisque j'ai élevé jusqu'à l'imago des larves prélevées dans les sédiments d'un petit ruisseau à cours lent, en basse altitude.

\section{SOUS-FAMILLE DES ERIOPTERINAE}

\section{TRIBU DES ERIOPTERINI}

\section{Genre Symplecta Meigen, 1830.}

6. S. (s. str.) hybrida (Meigen, 1804).

4 s. LG : $40 \mathrm{~m}, 3-\mathrm{V}$; Av : $220 \mathrm{~m}$, 19-VII et $2-\mathrm{VIII}$; Lz : $720 \mathrm{~m}, 8$ VIII. 


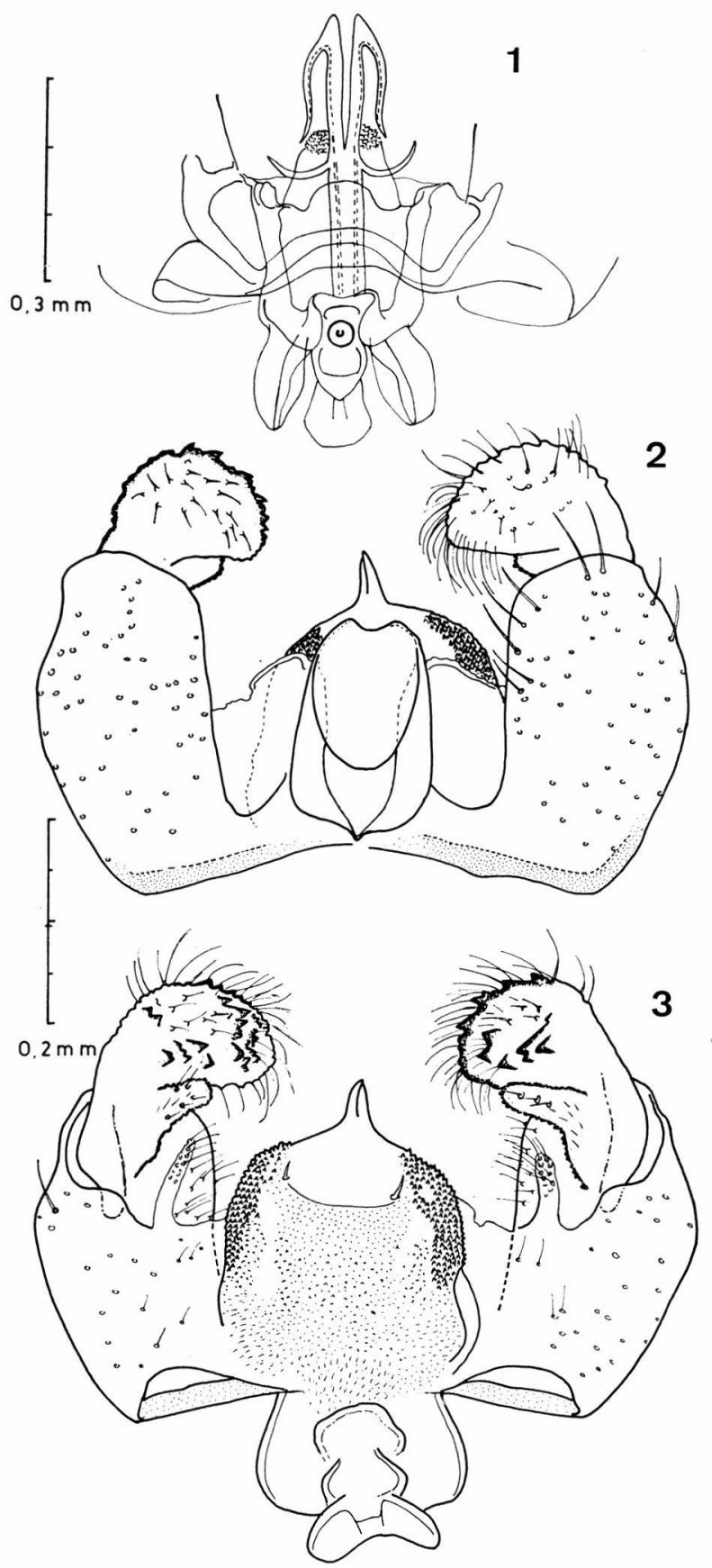

Fig. 1. - Ormosia (Rhypholophus) bifurcata ơ (Goetghebuer, 1920): édéage. FIG. 2 et 3. - Scleroprocta pentagonalis on (Loew, 1873): hypopyge, faces ventrale (fig. 2) et dorsale (fig. 3). 
Sous-genre Psiloconopa Zetterstedt, 1838.

** 7. S. (P.) stictica (Meigen, 1818).

3 คे, 1 \&. HP : $400 \mathrm{~m}, 29-\mathrm{III}$; HG : $190 \mathrm{~m}, 6-$ VIII et 4-IX.

Genre Scleroprocta Edwards, 1938.

* 8. S. pentagonalis (Loew, 1873).

7 ô, 1 ․ HP : 1180 à $470 \mathrm{~m}, 11$-VII à 8-VIII; Ar : $290 \mathrm{~m}, 25-\mathrm{V}$.

Genitalia $\hat{o}$ : fig. 2 et 3.

\section{TRIBU DES MOLOPHILINI}

Genre Ilisia Rondani, 1856.

** 9. I. (s. str.) occoecata Edwards, 1936.

1 s. HG : 440, 29-V.

Sous-genre Parilisia Savtshenko, 1976.

** 10. I. (P.) vicina (Tonnoir, 1920).

6 oิ. HP : $480 \mathrm{~m}, 18$-VII.

** 11. I. (P.) yezoana (Alexander, 1924).

(= czizeki Bangerter, 1947, selon Savtshenko et Krivolutskaya, 1976). 1 o. $\mathrm{Lz}: 530 \mathrm{~m}, 25-\mathrm{IV}$.

Genre Ormosia Rondani, 1856.

** 12. O. (s. str.) bicornis (De Meijere, 1919).

3 s. HG : $280 \mathrm{~m}, 3-\mathrm{X}$ et $190 \mathrm{~m}: 25-\mathrm{IX}$.

Genitalia 8 : fig. 4 et 5 .

** 13. O. (s. str.) bifida (Lackschewitz, 1940).

2 s. HP : $2230 \mathrm{~m}, 22$-IX et $1850 \mathrm{~m}, 6-\mathrm{VIII}$.

14. O. (s. str.) nodulosa (Macquart, 1826).

14 f. HP : 1550 à $1000 \mathrm{~m}, 13-$ VII à 10-VIII ; HG : $190 \mathrm{~m}, 25-\mathrm{V}$ à 27-VII.

Sous-genre Rhypholophus Kolenati, 1860.

** 15. O. (R.) bifurcata (Goetghebuer, 1920).

2 f, 1 ․ HG : $730 \mathrm{~m}, 15-\mathrm{X}$; Lz: $530 \mathrm{~m}, 26-\mathrm{IX}$.

Hypopyge partiellement dessiné par Goetghebuer et Tonnoir (1920).

Edéage : fig. 1.

Genre Molophilus Curtis, 1833.

** 16. M. bifidus Goetghebuer, 1920.

8 s. HP : 1180 à $470 \mathrm{~m}, 4$ à 8-VIII ; HG : $190 \mathrm{~m}, 25-\mathrm{V}$; Av : $260 \mathrm{~m}$, 1-VII.

Cette espèce peut désormais figurer dans la Limnofauna Europaea. J'en ai obtenu l'état imaginal par élevage de larves prélevées dans du sable, immergé en dehors des crues, bordant un cours d'eau lent (Av : $260 \mathrm{~m}$ ).

** 17. $M$. cinereifrons De Meijere, 1920.

2 ô. HP : $1280 \mathrm{~m}, 10-\mathrm{VIII}$; HG : $440 \mathrm{~m}, 29-\mathrm{V}$.

** 18. M. corniger De Meijere, 1920.

1 s. HP : $1180 \mathrm{~m}, 4-\mathrm{VIII}$. 
** 19. M. ochrescens Edwards, 1938.

1 s. Lz : 950 m, 25-VII.

** 20. M. pseudopropinquus Mendl, 1973.

1 s. HP : $480 \mathrm{~m}, 18-\mathrm{VII}$.

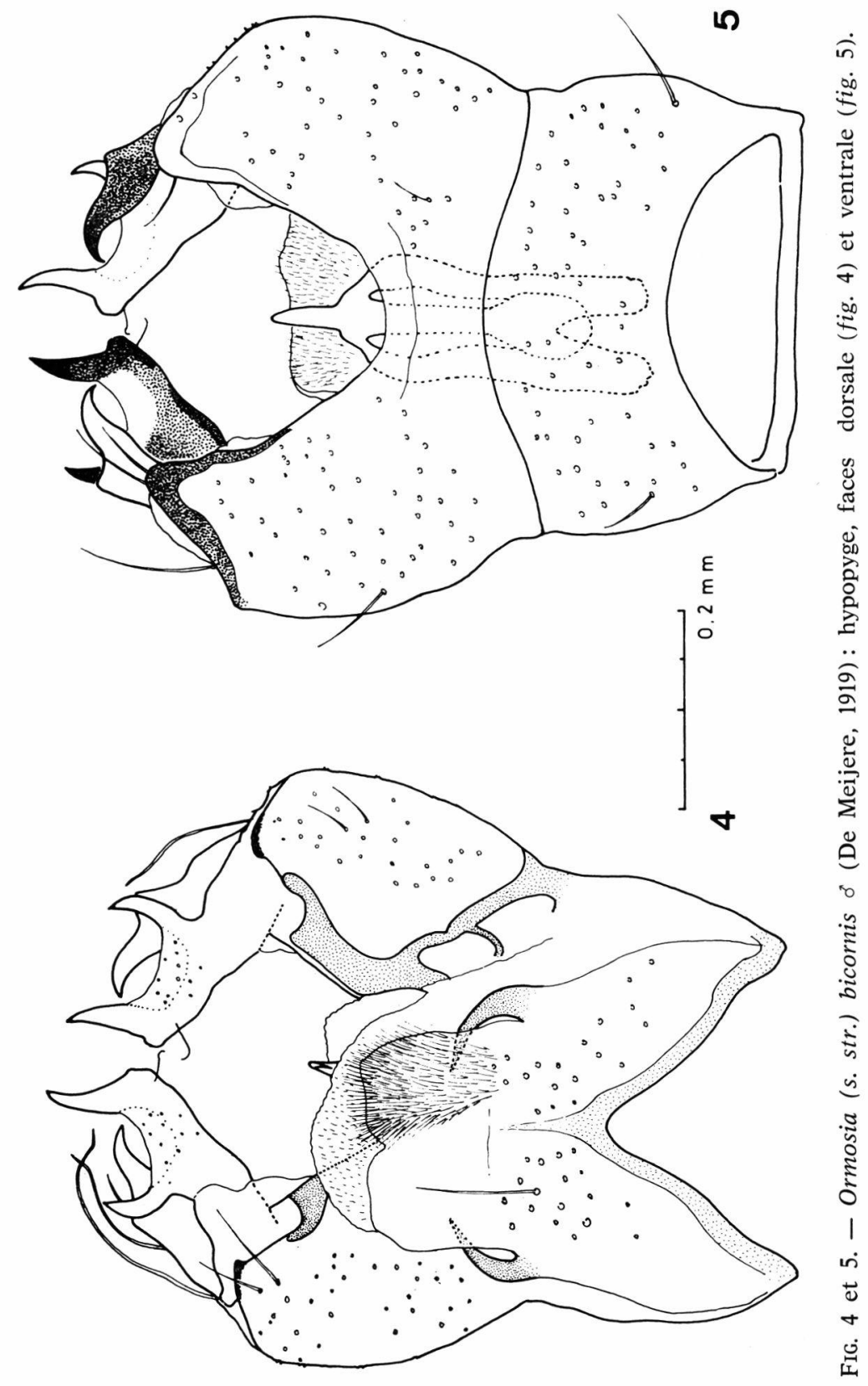




\section{TRIBU DES GONOMYINI}

Genre Idiocera Dale, 1842.

Souis-genre Protogonomyia Alexander, 1934.

21. I ( $P$ ) alboscutellata (Von Roser, 1840).

16 o, 2 o. HP : $1180 \mathrm{~m}, 4$-VIII et $1050 \mathrm{~m}, 6$ et 29-VII.

** 22. I. (P.) limbata (Von Roser, 1840).

63 ô, 7 q. HP : 1000 à $650 \mathrm{~m}, 11$-VII à 18-VIII.

Les larves de cette espèce étaient inconnues. Elles sont torrenticoles. En effet, j'ai pu observer que la ponte est déposée sur les pierres humides du lit de ruisseaux ombragés, à l'étiage. Les larves âgées se rencontrent dans le sable et les graviers bordant le cours.

Genre Gonomyia Meigen, 1818.

** 23. G. (s. str.) conoviensis Barnes, 1924.

1 s. Av : $350 \mathrm{~m}, 5$-VII.

** 24. G. (s. str.) lucidula De Meijere, 1920.

3 s, 1 ㅇ. Ar: $285 \mathrm{~m}, 12-\mathrm{VII}$; Lz: $1085 \mathrm{~m}, 6$-VII et 25-VIII.

\section{SOUS-FAMILLE DES LIMONIINAE}

\section{TRIBU DES ANTOCHINI}

\section{Genre Thaumastoptera Mik, 1866.}

* 25. T. calceata Mik, 1866.

1 ô. Ar : $290 \mathrm{~m}, 19-\mathrm{VII}$.

\section{TRIBU DES LIMONIINI}

\section{Genre Atypophthalmus Brunetti, 1911.}

$\because * 26$. A. inustus (Meigen, 1818).

2 ๙. HP: $960 \mathrm{~m}, 7$-VIII ; HG : $190 \mathrm{~m}, 4$-VIII.

\section{Genre Dicranomyia Stephens, 1829.}

** 27. D. (s. str.) luteipennis Goetghebuer, 1920.

2 o, 3 q. HP : 2150 et $1870 \mathrm{~m}, 15-\mathrm{IX}$ à 26-X.

Sous-genre Melanolimonia Alexander, 1965.

28. D. (M.) morio (Fabricius, 1787).

1 s.. HG : $190 \mathrm{~m}, 13-\mathrm{III}$.

** 29. D. (M.) stylifera Lackschewitz, 1928.

1 s. HP : $1250 \mathrm{~m}$, 7-VIII.

Sous-genre Sphaeropyga Savtshenko, 1976.

** 30. D. (S.) halterella Edwards, 1921.

1 ô. HP : $1870 \mathrm{~m}, 4-\mathrm{X}$.

Genre Metalimnobia Matsumura, 1911.

31. M. quadrimaculata (Linné, 1761).

1 s.. HP : $1000 \mathrm{~m}, 10$-VIII. 
Remarques.

1. La présence dans le Sud-Ouest de la France de l'Eriopterinae Lipsothrix errans (Walker, 1848), basée sur la citation d'une $q$ (Thomas, 1968), est confirmée : 1 o. HP, $1150 \mathrm{~m}, 13-\mathrm{VII}-1972$.

2. L'Eriopterinae Molophilus flavus Goetghebuer, 1920 a été obtenu à l'état imaginal par élevage. Biotope du prélèvement: Bryophytes aquatiques immergés dans un torrent, hors crue (HP: $1550 \mathrm{~m}, 2$ VII). L'espèce est à ajouter dans la Limnofauna Europaea.

3. La révision des Limoniidae paléarctiques par Lackschewitz et Pagast in Lindner (1940) ne comporte pas d'illustration des genitalia de Limonia flavipes. Les seules figures correspondant à cette espèce sont celles de Lackschewitz (1928) et Lackschewitz in Czizek (1931). Malheureusement, elles se révèlent inexactes: les paramères sont en réalité nettement plus courts et leur orientation ne correspond pas à celle des paramères des autres espèces figurées comparativement par cet auteur. A tel point que F. W. IEdwards a corrigé ultérieurement le tracé de Lackschewitz sur l'exemplaire de la bibliothèque du laboratoire d'Entomologie du British Museum (A. M. Hutson in litt.). Par suite, Limonia vaillanti Thomas, qui précisément était censé différer de $L$. flavipes par la forme des paramères, répond en fait au concept de L. flavipes des $\mathrm{D}^{\text {rs }}$ Starý et Savtshenko (J. Starý in litt.) ainsi qu'à celui d'Edwards (A. M. Hutson in litt.).

Limonia vaillanti Thomas, $1968=$ L. flavipes (Fabricius, 1787).

A ce propos, il faut également signaler que l'indication de Lackschewitz et Pagast (1940), concernant l'extrémité du pénis de $L$. flavipes " nicht zweihörnig, wie bie den anderen Arten der Gruppe " est, elle aussi, formellement inexacte.

Je remercie MM. J. Starý (Olomouc) qui a examiné un exemplaire de Limonia vaillanti et a attiré mon attention sur l'identité des deux espèces et A. M. Hutson (Londres) pour son aimable collaboration au niveau de la collection Edwards.

\section{PTYCHOPTERIDAE}

Genre Ptychoptera Meigen, 1803.

* 32. P. lacustris Meigen, 1830.

13 ㅅ, 3 ‥ HP : $1250 \mathrm{~m}, 13-\mathrm{VII}$; HG : $190 \mathrm{~m}, 20-25-\mathrm{V}, 25-\mathrm{VIII}, 25-$ IX ; T : $480 \mathrm{~m}, 24-\mathrm{VI}$.

Remarque.

Cette famille est à peu près ignorée des hydrobiologistes et les larves demeurent très mal connues. Brindle (1962) considère les larves des espèces de Grande-Bretagne comme semi-aquatiques et les signale dans des milieux d'eau stagnante. Peus in Illies (1967) indique 
pour toutes les espèces européennes, le seul milieu des sources (crénon). Dans le Sud-Ouest de la France, une espèce au moins $P$. paludosa Meigen, 1804 - peut vivre dans les ruisseaux ombragés à faible pente, très loin des sources.

\section{TRAVAUX CITÉS}

Brindele (A.). 1962. - Taxonomic Notes on the Larvae of British Diptera. 9. The family Ptychopteridae. Entomologist, 95 : 212-216.

Brindele (A.). 1967. - The Larvae and Pupae of the British Cylindrotominae and Limoniinae (Diptera, Tipulidae). Trans. Soc. Br. Ent., 17 (7) : 151-216.

BRINDLE (A.) et BRYCE (D.). 1960. - The Larvae of the British Hexatomini (Dipt., Tipulidae). Entomologist's Gaz., 11 : 207-224.

CzIzex (K.). 1931. - Die mährischen Arten der Dipterenfamilien Limoniidae und Cylindrotomidae. Cas. morav. zemsk. Mus., $28-29$ (1933): 289-495 + 1 pl.

Goetghebuer (M.) et Tonnoir (A.). 1920. - Catalogue raisonné des Tipulidae de Belgique. Bull. Soc. ent. Belg., $2: 131-147+2$ pl.

LACKSCHEWITZ (P.). 1928. - Die paläarktischen Limnobiiden (Diptera) des Wiener Naturhistorischen Museums. Annln naturh. Mus. Wien., $42: 195-244+2$ pl.

Lackschewitz (P.) et Pagast (F.). 1940. - 16. Limoniidae. In E. Lindner, Die Fliegen der palaearktischen Region. $135: 1-16+4$ pl.

Nielsen (P.). 1967. - Limoniidae. In J. Illies, Limnofauna Europaea. Stuttgart. $474 \mathrm{p}$.

Peus (F.). 1967. - Ptychopteridae, Chaoboridae, Dixidae. In J. Illies, Limnofauna Europaea. Stuttgart. 474 p.

Pierre (C.). 1924. - Diptères: Tipulidae. In Faune de France. 8. Paris, 159 p.

Savtshenko (E.) et Krivolutskaya (G. O.). 1976. - Diptères Limoniidae des Kouriles méridionales et du Sud de Sakhaline. Editions "La Pensée scientifique », Kiev, 160 p. (en russe).

Thomas (A.). 1968. - Limoniidae et Ptychopteridae du Sud-Ouest de la France (Diptera) (1 ${ }^{\text {re }}$ note). Annls Limnol., 4 (2) : 225-234.

Tromas (A.G. B.). 1972. - Sur quatre Limonia (Dicranomyia) européennes peu connues (Diptera, Tipulidae). Annls Limnol., 8 (1): 63-70. 